Introduction: Special Section

\title{
EDITORS' INTRODUCTION TO COMMENTARIES ON STATE REGULATION OF PSYCHOANALYSIS
}

Psychoanalysis, Culture \& Society (2007) 12, 50.

doi:10.1057/palgrave.pcs.2100113

I

n our last issue, 11/3, we published Jan De Vos's strong polemic against French and Belgian psychoanalysts' reactions to state moves to regulate psychoanalysis. De Vos critiqued French analysts, including JacquesAlain Miller, for, among other things, missing the historic opportunity to differentiate psychoanalysis from a scientistic and medicalized psychology. We also published Jacques China's review of a conference in the UK on the topic of state regulation of psychoanalysis. These papers revealed striking differences between the way analysts view the issue: differences, for example, between those who insist that psychoanalysis is not a mental health discipline at all, those who believe it is a mental health discipline but want to regulate its standards internally and keep the state out, and those who welcome state attempts to protect mental health "consumers" by creating standards of care and procedures for complaints. Given the importance of the topic in the history of psychoanalysis, and the widely disparate views, we invited three analysts to comment further on the issue. Laurel Bass Wagner and Ona Nierenberg take divergent views on recent events in New York State, where a group of psychoanalysts themselves lobbied for a state law to regulate the practice of psychoanalysis and were successful. Both Wagner and Nierenberg are critical of this move, but each formulates the stakes of the move quite differently. Ian Parker discusses the situation in the UK, focusing on the contentious issues of "evidence based practices" and complaints procedures. The issues are complex and of great importance to the future of psychoanalysis. We welcome further conversation on the topic.

Lynne Layton and Simon Clarke Brookline, MA, USA. 